\title{
Factores de riesgo asociados a epilepsia en escolares en el Hospital Regional del Norte
}

Risk factors associated with epilepsy in school children at the Regional Hospital of the North

Rubén Darío Acosta Zepeda, * Mario Velásquez.

\section{RESUMEN}

Antecedentes: La prevalencia activa de epilepsia en la edad escolar es de un $17.7 \%$ a nivel mundial. Se estima que entre el 80 al $85 \%$ de los pacientes afectados residen en países de bajos ingresos, donde hay poca disponibilidad de atención especializada. Objetivo: Definir los factores de riesgo asociado a epilepsia en escolares que acuden a la consulta externa de Neurología Pediátrica, Hospital Regional del Norte IHSS, en el periodo de enero 2018 a junio del 2018. Pacientes y Método Estudio Descriptivo Transversal cuantitativo con diseño no experimental, muestra no probabilística. Se aplicó una encuesta de 26 preguntas a 46 escolares de 6 años a 11 años con 364 días que asistieron a la consulta externa de Neurología Pediátrica del Hospital Regional del Norte. Resultados: El sexo masculino representó $63 \%$, el rango de edad de 6 a 9 años representó el $72 \%$. El antecedente materno de amenaza de aborto se presentó en el $23.9 \%$, el antecedente personal patológico de asfixia perinatal en un $26 \%$. La relación entre amenaza de aborto y asfixia perinatal se presentó en un $10.8 \%$. El $43 \%$ de los pacientes presentó el antecedente positivo de epilepsia familiar; el control adecuado de las crisis epilépticas se presentó en un $84.8 \%$. El medicamento Levetiracetam se presentó en el $52 \%$ de los casos; el estudio de electroencefalograma se realizó en el 100\% de

\footnotetext{
${ }^{*}$ Residente de tercer año del postgrado de Pediatría, UNAHVS

**Neurólogo-Pediatra-Epileptólogo del IHSS-RN.

Dirigir correspondencia a acostazruben89@gmail.com Recibido: 10 de Agosto del 2018 Aprobado: 20 de Septiembre 2018
}

los pacientes. Conclusiones: la identificación de estos factores puede contribuir a un diagnóstico más oportuno y manejo integral del escolar con epilepsia.

\section{PALABRAS CLAVES}

Epilepsia, factores de riesgo, Escolares

\section{ABSTRACT}

Background: The active prevalence of epilepsy in school age is $17.7 \%$ worldwide. It is estimated that between 80 and $85 \%$ of affected patients reside in low-income countries, where there is little availability of specialized care. Objective: Define the risk factors associated with epilepsy in schoolchildren attending the outpatient clinic of Pediatric Neurology, Hospital Regional of the North IHSS, in the period from January 2018 to June 2018. Patients and Method: Quantitative Cross-sectional Descriptive Study with non-experimental design nonprobabilistic sample. A survey of 26 questions was applied to 46 schoolchildren from 6 years to 11 years with 364 days who attended the outpatient clinic of Pediatric Neurology of the Hospital Regional of the North. Results: Male sex represented $63 \%$, the age range of 6 to 9 years represented $72 \%$. The maternal antecedent of threatened abortion was presented in $23.9 \%$, the personal pathological antecedent of perinatal asphyxia in $26 \%$. The relationship between the threat of abortion and perinatal asphyxia was $10.8 \% .43 \%$ of the patients presented a positive history of family epilepsy; appropriate control of epileptic seizures was presented in $84.8 \%$. The drug 
Levetiracetam was present in $52 \%$ of the cases; a electroencephalogram study was performed in $100 \%$ of the patients. Conclusions: the identification of these factors can contribute to a more timely diagnosis and comprehensive management of the school with epilepsy.

\section{KEYWORDS}

Epilepsy, risk factors, Schoolchildren

\section{INTRODUCCIÓN}

La epilepsia es un trastorno cerebral caracterizado por una predisposición persistente del cerebro para generar crisis epilépticas, caracterizadas por el conjunto de síntomas y signos transitorios, que se deben a una cantidad excesivamente anormal o sincrónica. La Liga Internacional Contra la Epilepsia (ILAE) la define como: una enfermedad del cerebro que presente por lo menos dos convulsiones no provocadas, que ocurren con una diferencia mayor de 24 horas, una convulsión no provocada y una probabilidad de crisis futuras similares al riesgo de recurrencia general después de dos ataques no provocados, que se producen durante los próximos 10 años $(1,2,3)$.

La prevalencia de la epilepsia activa en la edad escolar es de $17.7 \%$ a nivel mundial, no obstante, otras literaturas mencionan una prevalencia de 3.4 a 11.3 casos por cada mil individuos en la población infantil. $Y$ se afirma que sólo el $30 \%$ de las crisis epilépticas en la edad escolar son de etiología sintomática ${ }^{(4)}$. Entre el 80 a $85 \%$ de los pacientes afectados, residen en países de bajos y medianos ingresos, donde hay un limitado acceso a la atención especializada, pruebas diagnósticas apropiadas y suministro de medicamentos ${ }^{(5)}$.
La epilepsia se clasifica en 3 categorías: 1.Epilepsia Idiopática: se define como toda epilepsia de predominio genético u origen genético presunto y que no hay ninguna anormalidad neuroanatómica

neuropatológica. 2. Epilepsia Sintomática: es una epilepsia de causa genética o adquirida, asociada a anomalías anatómicas y características clínicas de enfermedad o afección subyacente. 3. Epilepsia Criptogénica: es definida como una epilepsia sintomática de naturaleza, la cual presume que no se ha identificado la causa, y que no puede ser catalogado como epilepsia sintomática ni como epilepsia idiopática $(3,6)$.

En el periodo de la infancia se agrupan las crisis febriles plus, la epilepsia occipital de la infancia de inicio temprano, la epilepsia en crisis mioclónicas atónicas, epilepsia de ausencia infantil, epilepsia benigna con puntas centrotemporales, epilepsia frontal nocturna autosómica dominante, epilepsia occipital de la infancia de inicio tardío, epilepsia con ausencias mioclónicas; la mayoría de estos síndromes epilépticos se asocian con un fuerte componente genético y en su mayor parte son de curso benigno y autolimitado $(7,8)$.

Dentro de los factores de riesgo más importantes se encuentran: 1. Prenatales: amenaza de aborto materna, preclamsia materna, infección del tracto urinario materna, malformaciones cerebrales durante el periodo embrionario. 2. Neonatales: encefalopatía hipóxico-isquémica, hemorragias intracraneales, contusiones cerebrales, alteraciones metabólicas y encefalopatías tóxicas. 3. Enfermedades infecciosas: meningitis, infecciones parenquimatosas, neurocisticercosis. 4. Traumatismos craneales. 5. Tumores cerebrales. 6. Eventos cerebrovasculares.7. Intoxicaciones: fármacos o herbicidas. 8. Factores genéticos. $(9,10,11)$ 
Dentro de las manifestaciones clínicas una crisis epiléptica, suele ser un evento clínico marcado por síntomas y signos de gran variabilidad; las crisis pueden afectar las funciones cognitivas, motoras o del comportamiento. Esta variedad de manifestaciones dependerá del sitio u origen de la descarga a nivel cerebral, o del comportamiento de su propagación $(1,12)$.

El diagnóstico de un paciente con epilepsia se basará en la realización de una adecuada historia clínica, enmarcada en una anamnesis detallada, una cuidadosa exploración física y unas pruebas complementarias. Dentro de ellas están las técnicas de neuroimagen como la tomografía cerebral y la resonancia magnética, siendo éste, el estudio de imagen de elección para pacientes con epilepsia. El electroencefalograma sigue siendo la herramienta diagnóstica más importante dentro de esta etapa escolar; ya que en su mayoría no presenta anomalías estructurales en el sistema nervioso central.

La decisión de iniciar el tratamiento debe adaptarse a cada niño. "una convulsión no es la epilepsia " o " EEG nunca debe ser tratado " es peligroso si se aplica de forma sistemática. La elección del fármaco dependerá de las características clínicas de la convulsión asociado con los resultados de estudios complementarios ${ }^{(13,14) \text {. }}$

El objetivo de este artículo es identificar cuáles son los factores de riesgo más importantes para el desarrollo de epilepsia en la edad escolar, realizando de esta manera medidas preventivas frente a estos factores de riesgo y creando además, políticas de seguridad para los pacientes de escasos recursos que tienen escasa disponibilidad de atención especializada.

\section{PACIENTES Y MÉTODOS}

Se realizó un estudio descriptivo trasversal, con enfoque cuantitativo y diseño no experimental. Con un muestreo no probabilístico a 46 pacientes escolares entre las edades de 6 años a 11 años con 364 días de ambos sexos con diagnóstico de epilepsia dentro del periodo escolar de la consulta externa de neurología pediátrica de la jornada matutina del Hospital Regional de Norte IHSS con autorización de sus padres de participar en el estudio.

Se excluyeron a todos los pacientes con diagnóstico de epilepsia no comprendido dentro del periodo escolar y todos aquellos pacientes que no desean participar en el estudio. Se recolectó datos por medio de la aplicación de una encuesta de 26 preguntas, 24 preguntas cerradas y 2 preguntas abiertas; donde se englobaron siete categorías: datos generales, factores socioeconómicos, antecedentes prenatales, antecedentes personales patológicos, datos familiares y tratamientos recibidos. Todas las encuestas se digitalizaron y analizaron bajo el programa estadístico SPSS versión 22 para Windows, donde se realizaron análisis descriptivos de distribución de frecuencias y tablas de contingencia.

\section{RESULTADOS}

Según el género se encontró en los escolares con epilepsia en la consulta externa de Neurología Pediátrica del HRNIHSS fue el masculino en el $63 \%$ (29) y el sexo femenino en el $37 \%$ (17) de los casos. Los rangos de edades se presentaron en: $71.7 \%$ (33) en el rango de edad de 6 a 9 años y en $28.3 \%$ (13) en el rango de edad de 10 a 11años con 364 días. La procedencia en el departamento de Cortés 
fue de: $76.4 \%$ (35). La raza mestiza se presentó en el $95.7 \%$ (44) de los casos.

Se determinó que la distribución de las patologías maternas durante el embarazo de los pacientes en un $67 \%(31)$ no presentó ninguna patología durante el embarazo, pero el antecedente de amenaza de aborto se presentó en un 24\%(11). (Ver Tabla 1)

De la distribución de antecedentes personales patológicos de los pacientes el $47.8 \%$ (22) de ellos no presentó ningún antecedente personal patológico; sin embrago el antecedente de asfixia perinatal se presentó en un $26.1 \%$ (12) de los casos. (Ver gráfico No.1) En relación al antecedente familiar de epilepsia se determinó que el $43.5 \%$ (20) de los pacientes presentó el antecedente familiar positivo para epilepsia. (Ver gráfico No. 2)

Dentro de la distribución de frecuencias del uso de medicamentos antiepilépticos de los pacientes, el levetiracetam se presentó en un $52.17 \%$ (24) de los pacientes, además el $78.2 \%$ (36) de los pacientes sólo utilizó un medicamento para el control de sus crisis epilépticas y el $84.8 \%$ (39) de los pacientes presentó un adecuado control de sus crisis epilépticas.

Se determinó que la distribución de estudios diagnósticos de los pacientes, el electroencefalograma se realizó en el $100 \%$ (46) de los pacientes, la tomografía cerebral en el $95.6 \%$ (44) de los pacientes, la resonancia magnética cerebral en el $30.4 \%$ (14) de los pacientes y los niveles de fármacos séricos en un $26 \%$ (12) de los pacientes. (Ver gráfico No. 3) Tabla No. 1: Antecedentes de patologías
maternas durante el embarazo

\begin{tabular}{|lcc|}
\multicolumn{1}{|c|}{ PATOLOGIA } & FRECUENCIA & $\%$ \\
\hline AMENAZA DE ABORTO & 11 & $24 \%$ \\
ITS & 0 & $0 \%$ \\
ITU & 0 & $0 \%$ \\
PRECLAMSIA & 4 & $9 \%$ \\
NINGUNO & 31 & $67 \%$ \\
\hline \multicolumn{2}{|c|}{ TOTAL } & $\mathbf{4 6 0} \%$ \\
\hline Fuente: Instrumento de recolección de datos de la investigación.
\end{tabular}

\section{Gráfico No. 1: Antecedente personal patológico}

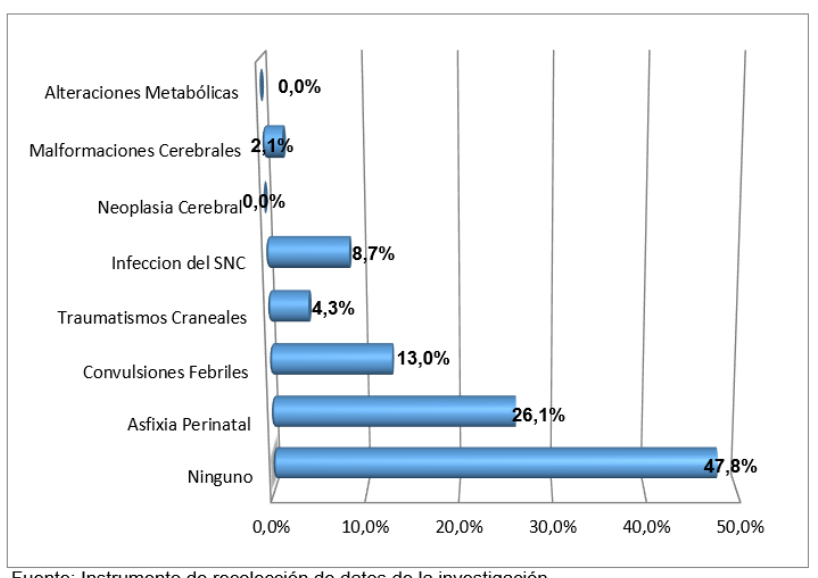

Fuente: Instrumento de recolección de datos de la investigación.

Gráfico No. 2: Antecedente familiar de epilepsia

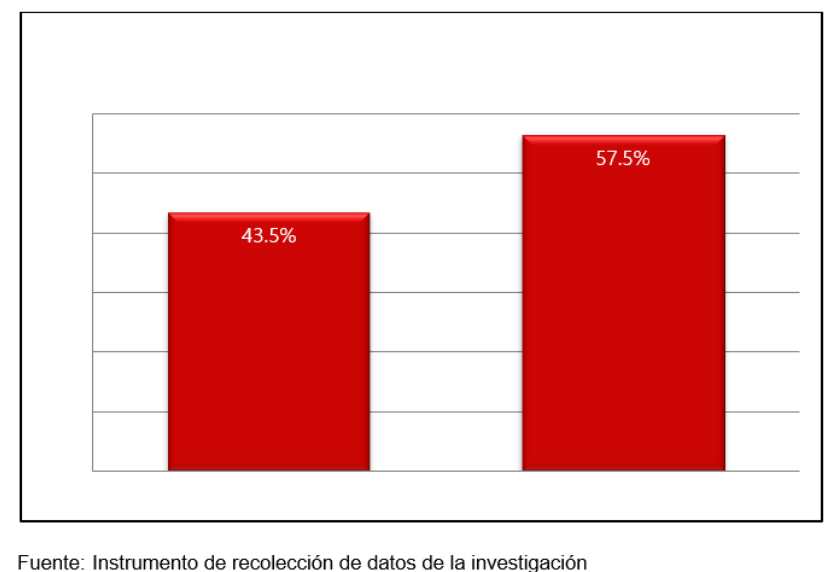




\section{Gráfico No. 2: Antecedente familiar de epilepsia}

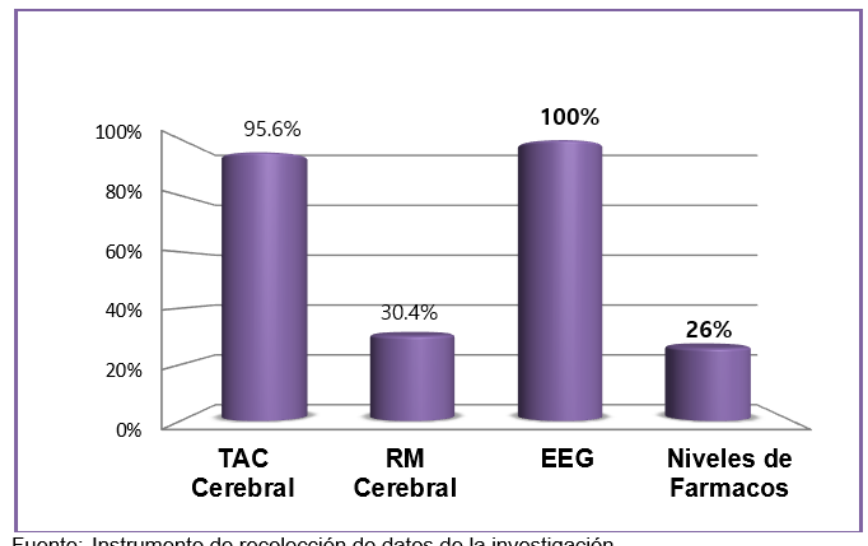

Fuente: Instrumento de recolección de datos de la investigación.

\section{DISCUSIÓN}

En nuestro estudio se encontró que el género más frecuente fue el sexo masculino en un $63 \%$ de los casos, similar a los estudios de Alsharif que reportó un porcentaje de $68.2 \%$ de epilepsia en escolares de sexo masculino(15). El rango de edad más frecuente fue el rango de 6 a 9 años de edad en un $72 \%$ de los casos, similar al estudio de Gómez y Pisón donde se reportó que la epilepsia dentro el rango de edad de 6 a 10 años fue de un $65.49 \%$. Lo que hace pensar que el cerebro del infante todavía es susceptible y está en proceso de desarrollo. Pero, cabe resaltar que la mayoría de crisis son autolimitadas y de curso benigno ${ }^{(16)}$.

Dentro de los antecedentes patológicos prenatales maternos más frecuentes encontramos que el antecedente de amenaza de aborto se presentó en el $24 \%$ de los casos, lo que difiere del estudio de Rozo e Izquierdo, el cual reportó un $12 \%$ de sus casos estudiados calificándolo como un factor de riesgo importante para el desarrollo de epilepsia, por lo que se recomienda a las madres un adecuado control prenatal durante la gestación ${ }^{(17)}$. En relación al antecedente personal patológico de los pacientes se encontró que el antecedente de asfixia perinatal se presentó en un $26 \%$ de los pacientes. Similar al estudio realizado por Cruz y Gallardo representando un $30 \%$ de sus pacientes en estudio con el antecedente positivo de asfixia perinatal, por lo que se determina que la asfixia perinatal se asocia significativamente al desarrollo de epilepsia en la infancia, ya que puede generar lesiones cerebrales altamente epileptogénicas ${ }^{(18) . \quad \text { Referente al }}$ antecedente familiar de epilepsia, representó en nuestro estudio que el $43 \%$ de los pacientes presentó el antecedente familiar positivo de epilepsia. Kind y Colaboradores encontraron un porcentaje similar a nuestro estudio en una población de escolares de 6 a 9 años en un $33 \%$.

En relación al control de la epilepsia se determinó que el control adecuado de las crisis epilépticas se presentó en un $84.8 \%$ de los pacientes que sólo utiliza un fármaco para el control de sus crisis epilépticas en un $78.2 \%$. En el estudio de Gómez y colaboradores, se presentó un adecuado control de crisis epilépticas en un $85 \%$ de la población en edad escolar, en general en esta edad la epilepsia suele ser benigna y la mayoría con evolución favorable con escasa refractariedad farmacológica(16). EI medicamento que con mayor frecuencia se utilizó como monoterapia o politerapia fue el Levetiracetam en el $52 \%$ de los pacientes. Lo que difiere del estudio lbekwe y colaboradores, reportaron que el fármaco más utilizado fue la Carbamazepina en un $39 \%{ }^{(19)}$. La evidencia formal del uso de Levetiracetam en niños es mínima y es potencialmente eficaz en la terapia de epilepsias benignas como las que se presentan en la edad escolar ${ }^{(20)}$. 
El método diagnóstico que representó la mayor frecuencia fue el Electroencefalograma en el $100 \%$ de los pacientes y además, el que reportó mayor frecuencia de anormalidades en un $37 \%$ de los pacientes. En lo reportado por Cruz y colaboradores es similar al estudio, ya que el $100 \%$ de sus casos se habían realizado el estudio de electroencefalograma, lo que resulta importante como herramienta diagnóstica de epilepsia en esta edad (18).

\section{CONCLUSIONES}

Se concluye que los factores de riesgo que se presentaron con mayor frecuencia fue: los pacientes del sexo masculino, los pacientes que se encuentran dentro del rango de edad de 6 a 9 años, el antecedente prenatal materno de amenaza de aborto, el antecedente personal patológico de asfixia perinatal y el antecedente positivo de un familiar con epilepsia. La identificación de estos factores de riesgo puede contribuir a realizar manejos e intervenciones oportunas en esta población escolar.

\section{CONFLICTO DE INTERESES}

Los autores manifiestan que durante la planeación y ejecución del presente trabajo de investigación no se presentó ningún conflicto de interés. 


\section{REFERENCIAS BIBLIOGRÁFICAS}

1. Gómez N. Diagnóstico de la epilepsia en la infancia, Revista Cubana de Pediatría, marzo 2009

2. Dura T. Epilepsia infantil en Navarra, An. Sanit. Navar.2007, vol. 30, N2 mayo- agosto

3. Fisher R. Practical clinical definitions of epilepsy, International League Againts Epilepsy, 2014. 55(4) 475-482

4. Saarinen M. Long-termchanges in the incidence of childhood epilepsy. A population study from Finland. Epilepsy \& Behavior 2016 81-85

5. Archana P. A pediatric epilepsy diagnostic tool for use in resource-limited settings: $A$ pilot study. Epilepsy \& Behavior 2016 (59) 57-61

6. Shorvon S. The etiologic classifications of epilepsy, International League Against Epilepsy.2011; 52(6) 1052-1057

7. Berg AT et al. Revised terminology and concepts for organization of seizures and epilepsies: report of the ILAE Commission on Classification and Terminology, 2005-2009. Epilepsy 2010; 51:676-685.

8. Berg Y. Terminología y conceptos revisados para la organización de crisis y epilepsias: informe de la comisión de la ILAE sobre clasificación y terminología 2005-2009

9. Miranda L. Factores de riesgo de epilepsia secundaria en niños, Revista de Neuropsiquiatría 2002:65 136-141

10. Cendes I. Aspectos genéticos de las epilepsias una visión actualizada, Rev Med Clinic 2013 24(6) 909-914
11. Otero D. La heterogeneidad genética en la epilepsia: presentación familiar, Revista Ciencias Médicas 2015: 19 (1) 141-150

12. Solari F. Crisis epilépticas en la población infantil, Rev. Med. Clin 2011 22(5) 647-654

13. Ryvlin P. Epilepsy surgery in children and adults, Lancet Neurology 2014: 13 1114-26

14. Jenny B. Pediatric epilepsy surgery surgery: couldage be a predictor of outcomes?, JNS pediatrics April 292016

15. Alsharif. M. Epilepsy as a health problems among schoool in Turaif, Northern Saudi Arabia, Electronic Physician ( ISSN: 2008-5842 ) August 2017, vol 9, Issue : 8 pages 5036-5042, DOI: 10.190825036

16. Gómez. L. Estudio de las epilepsias según la edad de inicio controladas durante 3 años en una unidad de neuropediatría de referencia regional, Anales de pediatría, ANPENDI 2013 NO. Of pág. 9

17. Rozo V. caracterización de los factores de riesgo de pacientes con epilepsia de difícil control en un hospital de cuarto nivel de Bogotá, Colombia. Acta neurológica de Colombia. 2014; 30 (4): 234-239.

18. Cruz. M. Factores asociados a epilepsia en niños en México: un estudio de caso control. Boletín médico del hospital infantil de México. 2017; 74(5): 334-340

19. Ibekwe R. Determinats of Noncompliance to clinic Appointments and Medications among Nigerian Children with Epilepsy: 
Experience in a Tertiary Health Facility in Enugu, Nigeria. Behavioral Neurology vol 2016 article ID: 6580416, 9 pag DOI: $10.1155 / 2016 / 6580416$.

20. Weijenber A. Levetiracetam Monotherapy in Children with Epilepsy: A systematic review. CNS DRUGS. MAY 2015 DOI: 10.1007/s40263-015-0248-9. 